\title{
Training the senior house officer: experience within a dermatology unit
}

\author{
Bart Ramsay
}

Department of Dermatology, Charing Cross Hospital, Fulham Palace Road, London W6 8RF, UK

If it is clear that undergraduate medical training needs redesigning, the same can be said for the postgraduate training of the senior house officer (SHO). ${ }^{1}$ Despite recent improvements, SHOs still work long hours. Much time is spent on service work like phlebotomy and clerical duties. Dissatisfaction with posts is common and few have a structured teaching programme built into their timetable. ${ }^{2}$ In particular, the hospital training of both the physician in training and the general practice trainee is not usually based on agreed objectives appropriate to their later career.,4 Teaching is usually peer-group led and tends to be on an ad hoc basis. To progress in hospital medicine one has to pass the M.R.C.P. For many trainees, attaining this diploma becomes a nightmare as they try to study during evenings when they are drained by the day's heavy service work and the energysapping nights on call.

By comparison, vocational general practice trainees have one contractual academic half day per week during their 2 year hospital attachment. During this period they absorb and develop the experiences gained in their hospital posts and apply them to the setting of general practice. They have small group teaching, formal lectures, tutorials, work on consultation technique and teaching on varied issues such as dealing with 'the angry patient' or 'how to give bad news'. In their final year in the training practice most general practice trainees have a full academic day release each week.

As a senior registrar in dermatology who has once been a vocational trainee in general practice, I have been able to compare training in general practice with that of hospital medicine. In contrast to general practice, where well-organized systematic training is common, I have observed many areas where the training of the medical SHO is deficient.

I have developed a training programme for SHOs which shows that this does not need to be

Correspondence: $\quad$ B. Ramsay, $\quad$ M.D., $\quad$ M.R.C.P., M.R.C.G.P.

Accepted: 29 January 1993 the case. The concepts for this programme arose from my own experience as a general practice vocational trainee. The approach that I describe is the minimum we should be providing in each hospital medical specialty and here I use dermatology as an example.

In the dermatology departments at Charing Cross and Central Middlesex Hospitals the SHO trainee is attached to the firm on a rotational scheme for 3 months and is embarked on a career in general practice or hospital medicine. On arrival the SHO is welcomed and the learning objectives for their attachment are defined. A curriculum (Table I) is used as a framework for formal teaching sessions. It is similar in structure to the log diary used by vocational trainees in general practice in the North West Thames region. ${ }^{5}$ In the firm's timetable a fixed period for formal teaching is set aside with the senior registrar for 50 minutes each week. Trainees are encouraged to add topics to this curriculum if they identify other areas they want to study.

There are three main training aims within the curriculum.

1. Knowledge. The teaching is adapted specifically to the trainee's needs. For those training for general practice more emphasis is given to those subacute and chronic conditions (Table I) which occur most frequently in general practice. For the physician in training much attention is given to common conditions and the cutaneous manifestations of systemic disease.

This knowledge is covered in a variety of ways. Dermatology is the most visual of all the specialties and in the formal teaching sessions slide projection, atlases and monographs are used to supplement experience gained in the clinic and from ward referrals. A therapeutic protocol for common skin conditions is being developed from the weekly sessions which will form a guiding framework for the future trainees. This is very important because of the short-term attachments and rapid turnover of junior posts. It gives the trainee a reference point in approaching problems from the first day in post. Developing this protocol is an education for the teacher as it demands an appraisal of the treat- 
Table I Dermatology curriculum

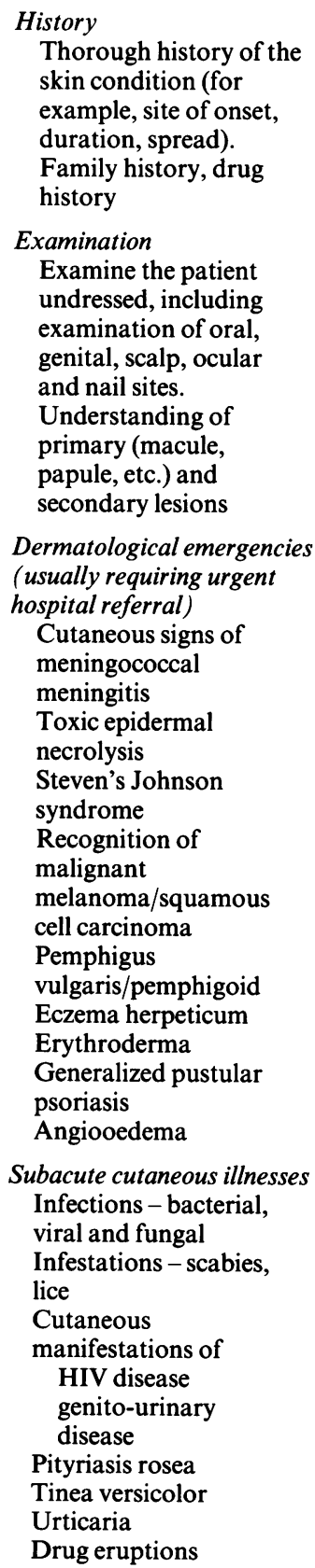

Chronic cutaneous diseases Eczema endogenous/exogenous Acne (vulgaris/rosacea) Psoriasis

Warts

Alopecia

Cutaneous manifestations of Internal malignancy Diabetes

Collagen vascular disease

Ultraviolet light and the skin

Cutaneous tumours

The genodermatoses

Lichen planus

Normal development Knowledge of benign congenital lesions

\section{Therapeutics}

Choice of base in

topical therapy

Emollients

Steroids

Dressings

Physical modalities

(UVR, cold point

cautery)

Environmental factors

(allergens, eczema

triggers)

Camofluage therapy

Simple remedies

Compliance and skin

treatments

Supporting the carer

(parent/companion of patient)

Patient associations

\section{Practical procedures}

Patch testing

Leg ulcer dressings

Skin scrapings for

mycology

Identifying the scabies mite

Cryotherapy

When, how and where to biopsy

Intralesional steroids

\section{Psychosocial}

Impact of skin disease on the patient

Awareness of patient understanding of their condition, expectations of treatment, fears including

'infectiousness' and inheritance of skin conditions

Myths of skin disease Implications of skin disease on employment ments used. The trainee is encouraged to study the conditions seen in clinic by reference to departmental dermatology texts which are provided by the consultants.

In-patient referrals from other departments are a treasure trove of both commonplace and rare conditions and the trainee can learn much from them. In addition, senior staff contribute to the educative process by bringing the trainee into their clinic rooms to see interesting patients thereby teaching on a wide range of conditions and therapies.

2. Practical skills. The second training goal is the acquisition of practical skills. Both the general practice trainee and physician in training gain from studying the curriculum sections on therapy and practical procedures (Table I).

A visit to the hospital pharmacy is valuable as one can see the immense variety of topical agents utilized and understand the implications for the patient of treatment with moisturisers, dithranol, tar and bandages. This is best taught in the hospital setting as most high street pharmacies do not have the broad range of formulations used in a dermatology unit.

There are video and audio tape programmes made by dermatologists teaching on common conditions and minor operative techniques. These are useful learning aids and can complement the teaching given by observing a senior colleague performing biopsies. In addition we have prepared an explanatory pamphlet for performance of biopsies. This is in order to help the trainee develop good biopsy technique and spare the patient the inconvenience of repeat biopsies related to poor initial sampling. Attendance at the weekly dermatopathology meetings enables the trainee to see whether the biopsy specimens taken were adequate. This also provides insight into the usefulness and limitations of histopathology. Weekly outpatient case note review of patients seen in clinic focuses on the why, where and when questions of investigation and therapy. This reduces unnecessary return clinic appointments as it helps guide the trainee's decision making.

3. Impact of skin disease on the patient. The third aim is to help the trainee appreciate what it is like to be a patient suffering from skin disease. Topics covered in this area are listed in the psychosocial section of the curriculum (Table I). It is important for a trainee to understand how skin disease causes disability and stigma for the patient. This will encourage them to develop consultation skills which identify these problems. These factors have major roles to play in patient's compliance with therapy, their social functioning and employment.

The requirements for teachers are a willingness to devote time to training the junior colleague, learning new teaching skills and a source of audio- 
visual aids. Without a formal training programme it is likely that as a result of service demands the educational requirements of the SHO trainee will not be met. A record is kept of sections studied in the trainee's curriculum and acts as a constant reminder of what has been covered within the formal sessions and as a spur to accomplish more. I have demonstrated that this programme works well in dermatology and is well appreciated by the SHOs. It can obviously be applied to all specialties. To set up a programme for other medical specialties, a curriculum list can be constructed using similar headings. Assistance should be sought from the regional organizer of general practice training to obtain advice on compiling subjects appropriate to general practice. Comparable help can be found for the physician in training from the Royal College of Physicians and the relevant specialty postgraduate advisors.

A major benefit of this programme is that it enhances the working relationship as enthusiasm generates more enthusiasm. A teaching ethos is passed on to the trainee. There is a positive environment created by the regular meetings which allows informal careers advice to be given if such a need is identified. Progress in the three training aims is assessed formally in a non-threatening way by a multiple slide test at the end of the attachment.

This curriculum approach is constantly evolving. The trainee's copy of the curriculum is given to them at the end of the 3 months attachment as not

\section{References}

1. Godfrey, R. Designing a doctor. All change? Lancet 1991, 338: 297-299.

2. Grant, J., Marsden, P. \& King, R.C. Senior house officers and their training. II. Perceptions of service and training. $\mathrm{Br} \mathrm{Med} \mathrm{J}$ 1989, 299: $1265-1268$.

3. Buckland, D. Training for the future. RCGP Connection 1991, 33: 8 .

4. Kearley, K. An evaluation of the hospital component of general practice vocational training. J R Coll Gen Pract 1990, 40: 409-414. all subjects are covered. This gives them a record of these lacunae for future study. A lot can be achieved in a short time.

The programme described here is one approach to making a relatively short attachment to a medical specialty an educational one for the physician in training or general practice trainee. Hospital medicine can learn much from innovative general practice day-release training which includes a structured postgraduate programme, small group learning, trainee log diary and opportunity to develop consultation skills. An academic half day each week in the contract of the physician in training is the first key step to foster genuine training for the largest hospital medical group after consultants. ${ }^{6}$ Why should the physician in training be disadvantaged in comparison to his or her general practice counterpart by not having contractual academic time? Hospital medical SHO training requires radical review. The benefits to both the hospital system and general practice would surely be considerable.

\section{Acknowledgement}

I would like to acknowledge the support and help of my senior colleagues, Drs Jonathan Leonard, Anne Powles, Chris Bunker and Jeff Cream, in promoting teaching for their junior doctors and the preparation of this paper. $\mathrm{Dr}$ Dan Veale has also given much advice and encouragement.

5. Styles, W. McN. General practice training in the hospital. $J R$ Coll Gen Pract 1990, 40: 401-402.

6. SCOPME Report. Improving the experience. Good practice in Senior house officer training. A report on local initiatives. September 1991. 